\title{
STUDY OF RADIATIVE DECAYS AND RELATED ChPT TESTS AT THE NA48/2 EXPERIMENT
}

\author{
STEFANO VENDITTI \\ Department of Physics, University of Pisa \& INFN, Pisa, Italy 56127 \\ FOR THE NA48/2 COLlaboration
}

\begin{abstract}
The latest results from the NA48/2 collaboration on charged kaon decays will be reviewed in this paper. The high statistics available, collected during 2003 and 2004 runs, allows the study of charged kaon decays up to branching ratios $(\mathrm{BR})$ of $\sim 10^{-8}$. The status of the analyses of several radiative decays $\left(\mathrm{K}^{ \pm} \rightarrow \pi^{ \pm} \pi^{0} \gamma, \mathrm{K}^{ \pm} \rightarrow \pi^{ \pm} \gamma \gamma, \mathrm{K}^{ \pm} \rightarrow \pi^{ \pm} e^{+} e^{-} \gamma\right.$ and $\mathrm{K}^{ \pm} \rightarrow \pi^{ \pm} e^{+} e^{-}$) will be discussed, including BR measurements and possible ChPT tests.
\end{abstract}

Keywords: Kaon decay; ChPT.

PACS numbers: $11.25 . \mathrm{Hf}, 123.1 \mathrm{~K}$

\section{Introduction}

The primary goal of the NA48/2 experiment, which took data in 2003 and 2004, was the measurement of the charged and neutral asymmetry in the $\mathrm{K}^{ \pm} \rightarrow \pi^{ \pm} \pi^{+} \pi^{-}$and $\mathrm{K}^{ \pm} \rightarrow \pi^{ \pm} \pi^{0} \pi^{0}$ decays $^{1}$. Along with these measurements, several other physics cases were considered and are still under investigation, some of which, as the cusp effect in the $\mathrm{K}^{ \pm} \rightarrow \pi^{ \pm} \pi^{0} \pi^{0}$ decay $^{2}$, were not even foreseen at the beginning of data-taking. This paper will deal with the ongoing radiative decay analyses, i.e. decays with a photon in the final state or which proceed through an internal photon conversion. These decays represent an important benchmark for theories aiming at precisely computing the hadronic part of the decay, among which one of the most credited in the kaon framework is Chiral Perturbation Theory (ChPT).

\section{The NA48/2 Beamline and Detectors}

A sketch of the NA48/2 beamline is shown in Fig. 1. Kaons are produced by impinging $400 \mathrm{GeV}$ protons from the SPS on a Beryllium target. Charged particles are selected through an achromat system in a momentum band centered at $60 \mathrm{GeV} / \mathrm{c}$ and with a r.m.s. of $3.8 \%$ and decay in the $\sim 113 \mathrm{~m}$ long fiducial region, downstream of which there are the detectors whose layout is shown in Fig. 2. 


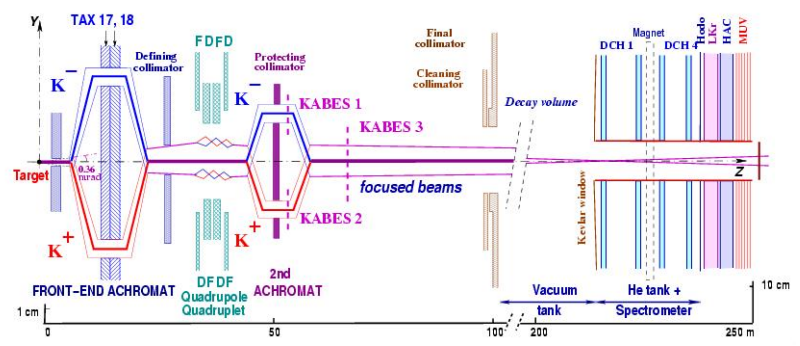

Fig. 1. Sketch of the NA48/2 beamline.

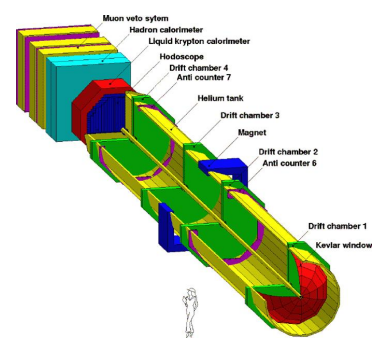

Fig. 2. Layout of the NA48/2 detectors.

The most important detectors are:

- the drift chamber (DCH) system, made of 4 octagonal DCHs and filled with a mixture of Argon(50\%) and Ethane(50\%). Each chamber is made of 4 views (each rotated by $45^{\circ}$ ) of 2 wire planes each, staggered by half wire pitch. A magnet, placed between DCH 2 and 3, gives the charged particles a $120 \mathrm{MeV} / \mathrm{c}$ transverse momentum kick. The resolution on the space point position is $\sim 100 \mu \mathrm{m}$, while the momentum resolution is:

$$
\frac{\sigma(P)}{P}=1.0 \% \oplus 0.044 \cdot P(G e V / c) \%
$$

- the charged hodoscope, placed between the last DCH and the calorimeter, made of two planes of 64 plastic scintillator strips. Its purpose is to yield time measurement for charged tracks and to provide fast trigger conditions. The time resolution obtained is $\sim 150 \mathrm{ps}$.

- the liquid krypton calorimeter (LKr), made of $132482 \times 2 \mathrm{~cm}^{2}$ cells, defined by $\mathrm{Cu}-\mathrm{Be}-\mathrm{Co} 127 \mathrm{~cm}$ long ribbons. The 27 radiation lengths and small Molière radius allow an excellent energy resolution:

$$
\frac{\sigma_{E}}{E}=\frac{0.032}{\sqrt{E(G e V)}} \oplus \frac{0.09}{E(G e V)} \oplus 0.0042
$$

Other detectors include a TPC detector (called Kabes) aiming at measuring the kaons' momentum and direction, a neutral hodoscope made of scintillating fibers, placed inside the LKr and mainly used as a control trigger, a system of 7 rings of iron and scintillator (called AKL) placed along the decay region, a hadronic calorimeter and a muon veto.

\section{3. $\mathrm{K}^{ \pm} \rightarrow \pi^{ \pm} \pi^{0} \gamma$ Decay}

The decay rate as a function of $\mathrm{W}=\sqrt{\left(P_{K} \cdot P_{\gamma}\right) /\left(M_{K} M_{\pi^{+}}\right)}$can be written as:

$$
\frac{d \Gamma}{d W} \simeq\left(\frac{d \Gamma}{d W}\right)_{I B}\left[1+2\left(\frac{m_{\pi}}{m_{K}}\right)^{2} W^{2}|E| \cos \left(\left(\delta_{1}-\delta_{0}\right) \pm \phi\right)+\left(\frac{m_{\pi^{+}}}{m_{K}}\right)^{4} W^{4}\left(|E|^{2}+|M|^{2}\right)\right]
$$




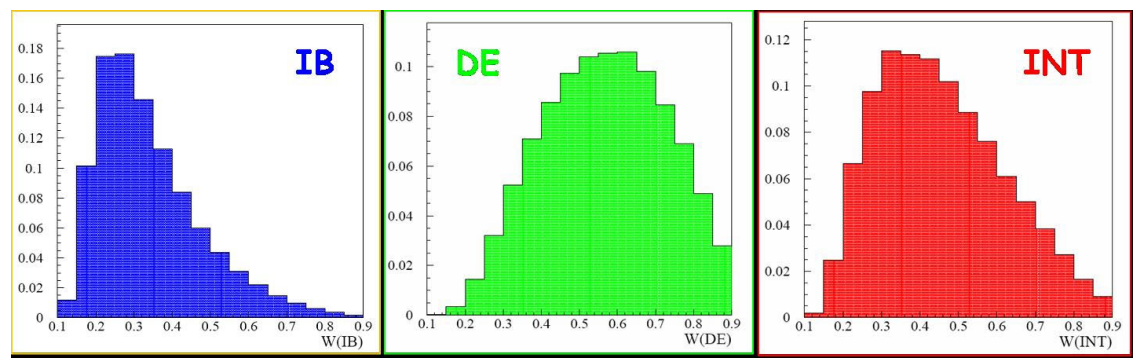

Fig. 3. W distributions for IB, DE and INT decays, respectively.

In the former expression three contributions can be distinguished, respectively representing the inner bremsstrahlung (IB), the direct emission (DE) and the interference (INT) part of the decay. $\mathrm{E}$ and $\mathrm{M}$ represent the electric and magnetic dipole moment of the decay. The INT part has never been measured so far: the INT and DE part estimation represent the main goal of this analysis. The selection of the $\mathrm{K}^{ \pm} \rightarrow \pi^{ \pm} \pi^{0} \gamma$ decay is performed requiring at least 1 track with $\mathrm{E} / \mathrm{p}^{\mathrm{a}}<0.85$, 3 clusters in the final state and performing acceptance cuts. Several BG channels were considered and reduced, e.g. by requiring the invariant mass of the selected particles to be compatible with the kaon mass; moreover the probability of photon mistagging was seen to be at $1 \%$ level. The residual $\mathrm{BG}$ is at $0.1 \%$ level of the DE part. In order to perform the DE and INT part measurement, the idea is to look at the $\mathrm{W}=\sqrt{\left(P_{K} \cdot P_{\gamma}\right) /\left(M_{K} M_{\pi}\right)}$ variable in the interval $0.2<\mathrm{W}<0.9$, where events from the IB, DE and INT parts distribute differently (see Fig. 3), and look for the weights minimizing the DATA/MC ratio. The fit function used for $\mathrm{W}$ is $\mathrm{W}_{D A T A}=(1-\mathrm{A}-\mathrm{B}) \cdot \mathrm{W}_{I B}+\mathrm{A} \cdot \mathrm{W}_{D E}+\mathrm{B} \cdot \mathrm{W}_{I N T}$ with A,B free parameters. The preliminary results on 2003 data are:

$$
\begin{gathered}
A_{D E}(0.2<W<0.9)=\left(3.35 \pm 0.35_{\text {stat }} \pm 0.25_{\text {syst }}\right) \% \\
A_{\text {INT }}(0.2<W<0.9)=\left(-2.67 \pm 0.81_{\text {stat }} \pm 0.73_{\text {syst }}\right) \%
\end{gathered}
$$

where the systematics are dominated by trigger efficiency. This represents the first measurement of the INT component of the decay so far.

\section{4. $\mathrm{K}^{ \pm} \rightarrow \pi^{ \pm} \gamma \gamma$ Decay}

The differential decay rate of the $\mathrm{K}^{ \pm} \rightarrow \pi^{ \pm} \gamma \gamma$ channel can be written as:

$$
\frac{d^{2} \Gamma}{d y d z}=\frac{m_{K^{+}}}{(8 \pi)^{3}}\left[z^{2}\left(|A+B|^{2}+|C|^{2}\right)+\left(y^{2}-\frac{1}{4} \lambda\left(1, r_{\pi}^{2}, z\right)\right)^{2}\left(|B|^{2}+|D|^{2}\right)\right.
$$

where $\mathrm{y}=\mathrm{P} \cdot\left(\mathrm{q}_{1}-\mathrm{q}_{2}\right) / \mathrm{M}_{K}^{2}, \mathrm{z}=\left(\mathrm{q}_{1}+\mathrm{q}_{2}\right) / \mathrm{M}_{K}^{2}=\mathrm{M}_{\gamma \gamma}^{2} / \mathrm{M}_{K}^{2}$ and $\mathrm{P}, \mathrm{q}_{1}, \mathrm{q}_{2}$ are respectively the pion and the two photon four-momenta. A, B, C and D only depend on $\mathrm{z}$ and

a $\mathrm{E} / \mathrm{p}$ is the ratio between the energy measured by the LKr and momentum measured by the DCHs 
on the free parameter $\hat{c}$, which is a function of several strong and weak coupling constants $^{3}$. Depending on the $\hat{c}$ value, the BR theoretical estimation, as well as the shape of the $\mathrm{m}_{\gamma \gamma}$ distribution, sizably change, as shown in Figs. 4 and 5. Corrections at $\mathcal{O}\left(\mathrm{p}^{6}\right)$ order in ChPT can increase BR by 30-40\%, depending on the $\hat{c}$ value.

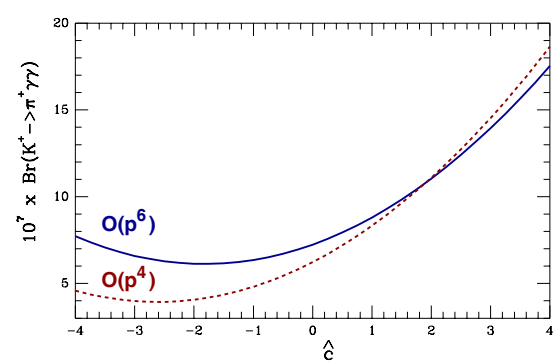

Fig. 4. $\mathrm{K}^{ \pm} \rightarrow \pi^{ \pm} \gamma \gamma \mathrm{BR}$ as a function of $\hat{\mathrm{c}}$ variable at $\mathcal{O}\left(\mathrm{p}^{4}\right)$ and $\mathcal{O}\left(\mathrm{p}^{6}\right)$ order of ChPT.

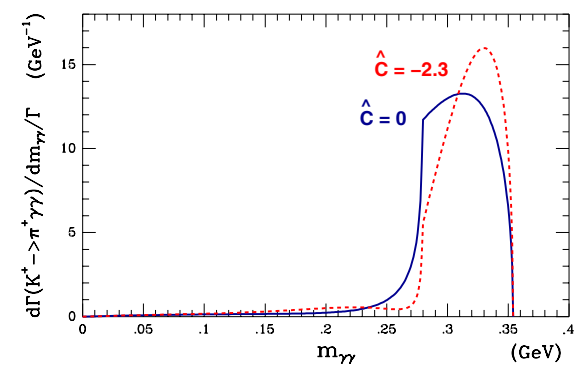

Fig. 5. Shape of the $\mathrm{m}_{\gamma \gamma}$ distribution at $\hat{\mathrm{c}}=0$ and -2.3 .

The event selection is performed by requiring at least one track with $\mathrm{E} / \mathrm{p}<0.8$ and two clusters and performing standard acceptance cuts. The invariant mass of the 3 final particles is required to be within $\pm 0.02 \mathrm{GeV} / \mathrm{c}^{2}$ from the kaon nominal mass, while the $\mathrm{m}_{\gamma \gamma}$ mass must be in the kinematic boundary $0<\mathrm{m}_{\gamma \gamma}<\mathrm{m}_{K^{+-}}$ $\mathrm{m}_{\pi^{+}}$. At the end 1164 events are selected, with $\sim 3 \%$ estimated BG, mostly from $\mathrm{K}^{ \pm} \rightarrow \pi^{ \pm} \pi^{0} \gamma$ decay. Comparisons between data and MC for reconstructed $\mathrm{K}$ mass and $\mathrm{m}_{\gamma \gamma}$ mass are shown in Figs. 6 and 7 . The $\hat{\mathrm{c}}$ value used for MC generation is 2.0 , which yields a good agreement with data.

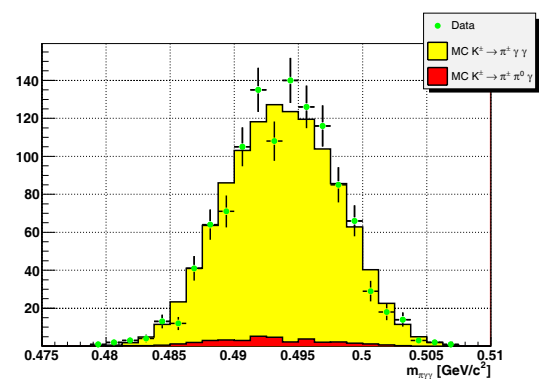

Fig. 6. Distribution of reconstructed kaon mass for $\mathrm{K}^{ \pm} \rightarrow \pi^{ \pm} \gamma \gamma$ events.

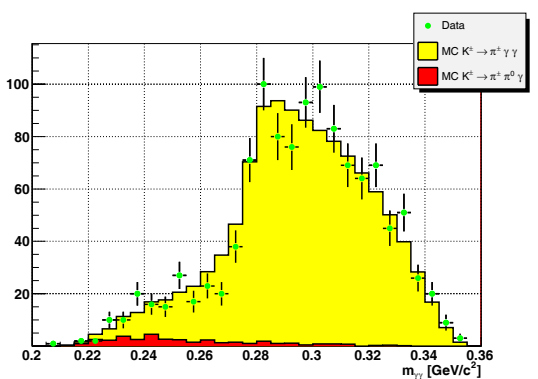

Fig. 7. Distribution of reconstructed $\mathrm{m}_{\gamma \gamma}$ mass for $\mathrm{K}^{ \pm} \rightarrow \pi^{ \pm} \gamma \gamma$ events.

The preliminary $\mathrm{BR}$ result, using $\mathrm{K}^{ \pm} \rightarrow \pi^{ \pm} \pi^{0}$ to compute the flux, is:

$B R\left(K^{ \pm} \rightarrow \pi^{ \pm} \gamma \gamma\right) / B R\left(K^{ \pm} \rightarrow \pi^{ \pm} \pi^{0}\right)=\left(1.07 \pm 0.04_{\text {stat }} \pm 0.08_{\text {syst }}\right) \cdot 10^{-6}$

Future tasks include the $\hat{c}$ measurement using a model-independent BR estimation. 


\section{5. $\mathbf{K}^{ \pm} \rightarrow \pi^{ \pm} e^{+} e^{-\gamma}$ Decay}

The theory of the $\mathrm{K}^{ \pm} \rightarrow \pi^{ \pm} e^{+} e^{-} \gamma$ and $\mathrm{K}^{ \pm} \rightarrow \pi^{ \pm} \gamma \gamma$ are very similar, as one can obtain the former from the latter by an internal $\gamma \rightarrow e^{+} e^{-}$conversion; naively one expects $\mathrm{BR}\left(\mathrm{K}^{ \pm} \rightarrow \pi^{ \pm} e^{+} e^{-} \gamma\right) \simeq 2 \alpha \cdot \mathrm{BR}\left(\mathrm{K}^{ \pm} \rightarrow \pi^{ \pm} \gamma \gamma\right)$. The $\mathrm{BR}$ is again a function of the $\hat{c}$ parameter, its value is enhanced by higher-order ChPT terms (Fig. 8) and predicted to be in the range $(0.9-1.6) \cdot 10^{-84}$. The goal is to perform a modelindependent $\mathrm{BR}$ measurement, use it to obtain an estimation of the $\hat{c}$ parameter and use this value to perform a model-dependent BR measurement.

The selection requires at least 3 tracks with total charge $= \pm 1$, at least 1 cluster not associated to the track, E/p $>0.94$ for $\mathrm{e}^{ \pm}$and $<0.8$ for $\pi^{ \pm}$and total energy between 54 and $66 \mathrm{GeV}$. BG suppression cuts include a cut on e- $\gamma$ angle to reject $\mathrm{K}^{ \pm} \rightarrow \pi^{ \pm} e^{+} e^{-}$decays (see Fig. 9), a cut on $\mathrm{e}^{+}-\mathrm{e}^{-}-\gamma$ invariant mass $\left(\mathrm{m}_{e e \gamma}<260\right.$ $\mathrm{MeV} / \mathrm{c}^{2}$, Fig. 10) and the kaon mass constraint $\left(480 \mathrm{MeV} / \mathrm{c}^{2}<\mathrm{M}_{e е \pi \gamma}<505\right.$ $\mathrm{MeV} / \mathrm{c}^{2}$, Fig. 11); in the latter two figures all the BGs considered are also shown.

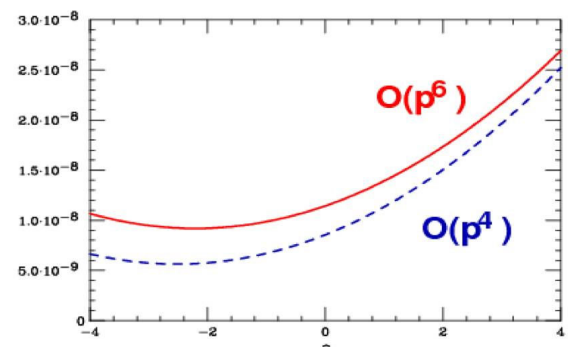

Fig. 8. $\quad \mathrm{K}^{ \pm} \rightarrow \pi^{ \pm} e^{+} e^{-} \gamma \mathrm{BR}$ value as a function of $\hat{c}$, at $\mathcal{O}\left(\mathrm{p}^{4}\right)$ and $\mathcal{O}\left(\mathrm{p}^{6}\right)$ order in ChPT.

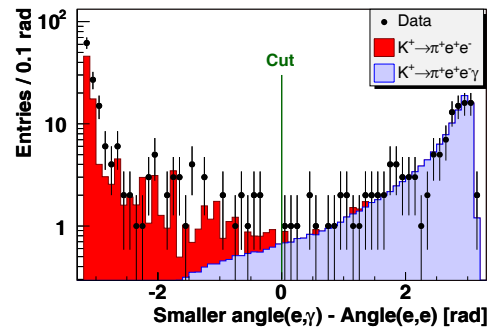

Fig. 9. Angle between electron and photon for $\mathrm{K}^{ \pm} \rightarrow \pi^{ \pm} e^{+} e^{-} \gamma$ and $\mathrm{K}^{ \pm} \rightarrow \pi^{ \pm} e^{+} e^{-}$events.

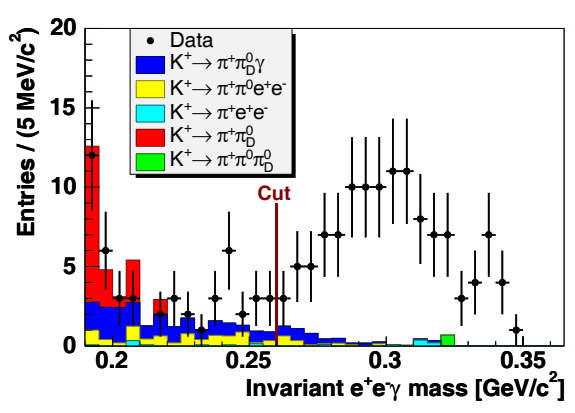

Fig. 10. $\mathrm{m}_{\text {ee }}$ distribution for $\mathrm{K}^{ \pm} \rightarrow$ $\pi^{ \pm} e^{+} e^{-} \gamma$ events.

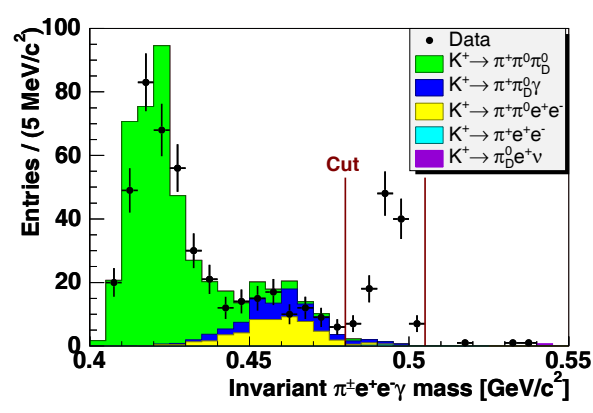

Fig. 11. $\mathrm{m}_{e e \pi \gamma}$ distribution for $\mathrm{K}^{ \pm} \rightarrow$ $\pi^{ \pm} e^{+} e^{-} \gamma$ events.

120 candidate events were selected, with an estimated BG of $7.3 \pm 1.7$ events. The channel used to compute kaon flux is $\mathrm{K}^{ \pm} \rightarrow \pi^{ \pm} \pi_{D}^{0}\left(\sim 14 \cdot 10^{6}\right.$ events), with 
$\pi_{D}^{0} \rightarrow e^{+} e^{-} \gamma$. A model-independent (MI) BR measurement was performed in $\mathrm{M}_{e e \gamma}$ bins with $5 \mathrm{MeV}$ steps, yielding:

$$
B R_{M I}\left(m_{e e \gamma}>260 M e V / c^{2}\right)=\left(1.19 \pm 0.12_{\text {stat }} \pm 0.04_{\text {syst }}\right) \cdot 10^{-8}
$$

A least square fit to extract $\hat{\mathrm{c}}$ yielded $\hat{\mathrm{c}}=0.9 \pm 0.45$. The result of the modeldependent (using $\hat{\mathrm{c}}=0.9$ ) $\mathrm{BR}$ value is:

$$
B R_{M I}=\left(1.29 \pm 0.13_{\exp } \pm 0.03_{\hat{\mathrm{c}}}\right) \cdot 10^{-8}
$$

All the results exposed have been published ${ }^{5}$.

\section{6. $\mathbf{K}^{ \pm} \rightarrow \pi^{ \pm} e^{+} e^{-}$Decay}

The $\mathrm{K}^{ \pm} \rightarrow \pi^{ \pm} e^{+} e^{-}$decay proceeds through suppressed FCNC processes, with an internal conversion of a virtual photon in an $\mathrm{e}^{+}-\mathrm{e}^{-}$pair. Its differential decay rate can be written as:

$$
\frac{d \Gamma}{d z}=P(z) \cdot|W(z)|^{2}
$$

where $|\mathrm{W}(\mathrm{z})|^{2}$ is the squared matrix element, $\mathrm{P}(\mathrm{z})$ is a phase space factor and $\mathrm{z}=\mathrm{M}_{e e}^{2} / \mathrm{M}_{K}^{2}$ is the only relevant kinematic variable (the other one is integrated). The goals of the analysis are a model-independent (in the accepted kinematic range) and a model-dependent BR estimation using three different parametrization of $\mathrm{W}(\mathrm{z})$ : polynomial and from 2 ChPT models ${ }^{67}$. The channel used to compute the kaon flux is $\mathrm{K}^{ \pm} \rightarrow \pi^{ \pm} \pi_{D}^{0}$. Selection cuts include requirement of a vertex compatible with 3 tracks, $\mathrm{E} / \mathrm{p}<0.85$ for $\pi^{ \pm}$and $>0.95$ for $\mathrm{e}^{+}$and $\mathrm{e}^{-}$. BG reduction cuts include cut on $\mathrm{e}^{+}-\mathrm{e}^{-}$invariant mass $\mathrm{M}_{e e}<140 \mathrm{MeV} / \mathrm{c}^{2}$ and on kaon mass (Fig. 12); BG events can be estimated directly on data using wrong-sign events, i.e. events whose total charge is wrong, which can be shown to be proportional to right-sign events (real BG). 7103 candidates were selected, with a residual BG of $\sim 0.8 \%$. A comparison between data and $\mathrm{MC}$ for the angle between $\mathrm{e}^{+}$and $\pi^{ \pm}$in $\mathrm{e}^{+}-\mathrm{e}^{-}$rest frame is shown in Fig. 13.

A fit in the $\mathrm{z}$ variable using parametrizations from the 3 tested model shows (Fig. 14) a reasonable agreement with data for all of them.

A model-independent $\mathrm{BR}$ was computed in the interval $0<\mathrm{z}<0.5$ :

$$
B R_{M I}=\left(2.26 \pm 0.03_{\text {stat }} \pm 0.03_{\text {syst }} \pm 0.06_{\text {ext }}\right) \cdot 10^{-7}=(2.26 \pm 0.08) \cdot 10^{-7}
$$

Model-dependent BRs can be computed for the 3 models and combined, adding an uncertainty due to the model dependence:

$$
B R=\left(3.08 \pm 0.04_{\text {stat }} \pm 0.04_{\text {syst }} \pm 0.08_{\text {ext }} \pm 0.07_{\text {model }}\right) \cdot 10^{-7}=(3.08 \pm 0.12) \cdot 10^{-7}
$$




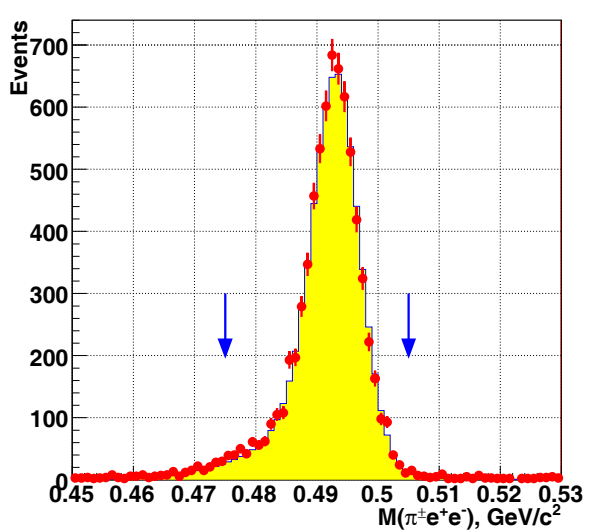

Fig. 12. Reconstructed kaon mass distribution for $\mathrm{K}^{ \pm} \rightarrow \pi^{ \pm} e^{+} e^{-}$events.

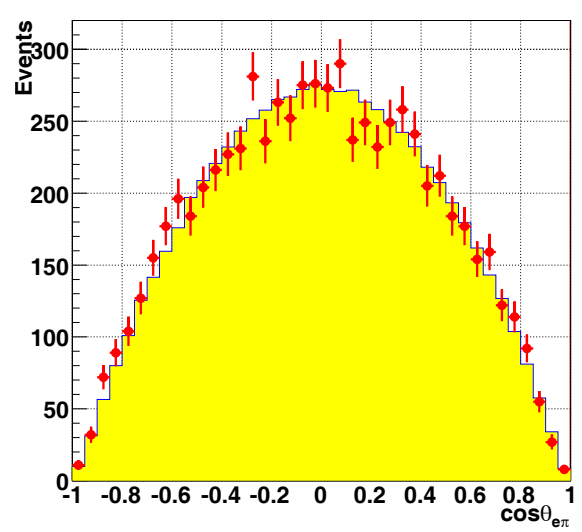

Fig. 13. Angle between $\mathrm{e}^{+}$and $\pi^{ \pm}$in $\mathrm{e}^{+}-\mathrm{e}^{-}$ rest frame.

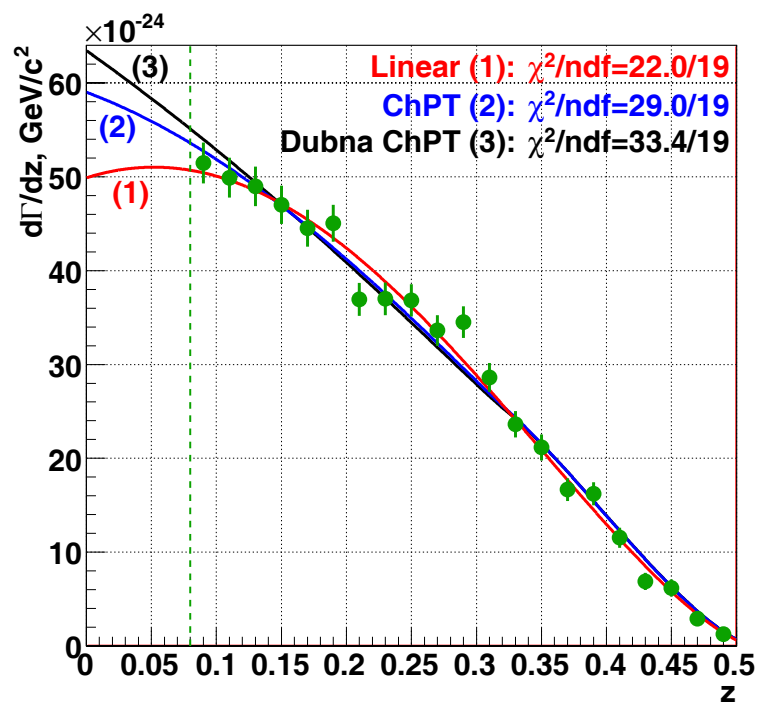

Fig. 14. Fit of experimental data using the 3 parametrizations for the differential decay rate.

\section{References}

1. J.R. Batley et al., Eur. Phys. Jour. C 52, 875 (2007).

2. J.R. Batley et al., Phys. Lett. B 633, 173 (2005).

3. G. D'Ambrosio, J. Portolés, Phys. Lett. B 386, 403 (1996).

4. F. Gabbiani, Phys. Rev. D 59, 094022 (1999).

5. J.R. Batley et al., Phys. Lett. B 659, 493 (2008).

6. D'Ambrosio et al., Jour. High En. Phys. 8, 4 (1998).

7. A.Z.Dubnickova et al., ArXiv:hep-ph/0611175. 